\title{
Conditionally Positive Definite Hyperfunctions
}

\author{
By \\ Jaeyoung ChUnG* and Dohan KIM**
}

\begin{abstract}
Making use of the heat kernel method we define a conditionally positive definite hyperfunction and obtain a Bochner-Schwartz type theorem for hyperfunctions which generalizes both the Bochner-Schwartz type theorem for distributions of GelfandVilenkin in $[\mathrm{GV}]$ and our version of Bochner-Schwartz theorem for (Fourier) hyperfunctions in [CCK2, CK1].
\end{abstract}

\section{$\S 1$. Introduction}

Generalizing the Bochner theorem which states that every positive definite function is the Fourier transform of a positive finite measure L. Schwartz proved the Bochner-Schwartz theorem for the distributions and tempered distributions, which states that every positive definite (tempered) distribution $u$ is the Fourier transform of a positive tempered measure $\mu$. In other words, $u$ can be expressed as

$$
\langle u, \varphi\rangle=\int \hat{\varphi}(\xi) d \mu(\xi), \quad \varphi \in C_{c}^{\infty} .
$$

Here, a positive measure $\mu$ is said to be tempered if $\int\left(1+|x|^{2}\right)^{-p} d \mu<\infty$ for some $p \geq 0$.

The above Bochner-Schwartz theorem was generalized in [CK1] for the space of Fourier hyperfunctions, which states that every positive definite Fourier hyperfunction $u$ is the Fourier transform of a positive measure $\mu$ of infraexponential type. Here, a positive measure $\mu$ is said to be of infra-exponential type if $\int e^{-\epsilon|x|} d \mu<\infty$ for every $\epsilon>0$.

Communicated by T. Kawai. Received April 18, 2002. Revised February 16, 2004.

2000 Mathematics Subject Classification(s): 42A82, 46F05.

*Department of Mathematics, Kunsan National University, Kunsan 573-701, Korea.

** Department of Mathematics, Seoul National University, Seoul 151-747, Korea. 
The concept of positive definite distributions has been generalized to the conditionally positive definite distributions (see Section 3 for the definition) which arise in the theory of generalized random process and the BochnerSchwartz theorem was generalized subsequently for conditionally positive distributions as follows.

Theorem 1.1 [GV]. Every conditionally positive definite (tempered) distribution $u$ of order $s$ can be expressed as

$$
\langle u, \varphi\rangle=\int_{\mathbb{R}_{0}^{n}}\left(\hat{\varphi}(x)-\alpha(x) \sum_{|k|=0}^{2 s-1} \frac{\hat{\varphi}^{(k)}(0)}{k !} x^{k}\right) d \mu(x)+\sum_{|k|=0}^{2 s} a_{k} \frac{\hat{\varphi}^{(k)}(0)}{k !}
$$

for all $\varphi \in C_{c}^{\infty}$. Here $\mu$ is a positive measure on $\mathbb{R}_{0}^{n}:=\mathbb{R}^{n}\{0\}$ satisfying

$$
\int_{0<|x| \leq 1}|x|^{2 s} d \mu(x)<\infty, \quad \int_{|x| \geq 1}(1+|x|)^{-p} d \mu(x)<\infty
$$

for some $p \geq 0$; (i) $\alpha(x) \in \widehat{C}_{c}^{\infty}$, (ii) $\alpha(x)-1$ has a zero of order $2 s+1$ at $x=0$ and (iii) the $a_{k}^{\prime} s$ are the complex numbers such that the $a_{k}^{\prime} s$ with $|k|=2 s$ satisfies the following

$$
\sum_{|i|=|j|=s} a_{i+j} \xi_{i} \bar{\xi}_{j} \geq 0
$$

for all complex numbers $\xi_{j} \in \mathbb{C},|j|=s$ and the $a_{k}^{\prime} s$ with $|k| \leq 2 s-1$ are certain complex numbers.

Generalizing the above Bochner-Schwartz type theorem for (tempered) distributions, we proved in [CCK3] the Bochner-Schwartz type theorem for Fourier hyperfunctions which states that every conditionally positive Fourier hyperfunction $u$ of order $s$ has the form (1.2), where the positive measure $\mu$ satisfies

$$
\int_{0<|x| \leq 1}|x|^{2 s} d \mu(x)<\infty, \quad \int_{|x| \geq 1} e^{-\varepsilon|x|} d \mu(x)<\infty
$$

for every $\varepsilon>0 ; \alpha(x)$ is the function

$$
\alpha(x)=e^{-|x|^{2}} \sum_{|k|=0}^{s} \frac{1}{k !} x^{2 k}
$$

and the $a_{k}^{\prime}$ s with $|k|=2 s$ have the same properties as in Theorem 1.1.

This result is also a generalization of the Bochner-Schwartz theorem for Fourier hyperfunctions in [CK1]. 
The concept of positive definiteness and conditionally positive definiteness can be naturally defined in the space of Fourier hyperfunctions but not in the space of hyperfunctions since a hyperfunction is defined locally as an analytic functional but not globally. In [CCK2] by applying the heat kernel method of T. Matsuzawa positive definite hyperfunctions have been introduced in terms of the Gauss transform and it is proved that positive definite hyperfunction is just a positive definite Fourier hyperfunction.

In this paper, making use of the heat kernel method we define the conditionally positive definite hyperfunction and obtain a Bochner-Schwartz type theorem which generalizes both Theorem 1.1 and the Bochner-Schwartz theorem for hyperfunctions in [CCK2]. As a main result we prove that every conditionally positive definite hyperfunction is nothing but a conditionally positive definite Fourier hyperfunction and also obtain an equivalent condition in terms of their Gauss transforms.

\section{§2. Heat Kernel Approach to (Fourier) Hyperfunctions}

In this section we introduce briefly the spaces of Fourier hyperfunctions and hyperfunctions and represent (Fourier) hyperfunctions as the initial values of solutions of the heat equation, which we call the heat kernel method.

Here we use the multi-index notations for $x=\left(x_{1}, \ldots, x_{n}\right) \in \mathbb{R}^{n}, \alpha=$ $\left(\alpha_{1}, \ldots, \alpha_{n}\right) \in \mathbb{N}_{0}^{n}:|\alpha|=\alpha_{1}+\cdots+\alpha_{n}, \alpha !=\alpha_{1} ! \ldots \alpha_{n} !, x^{\alpha}=x_{1}^{\alpha_{1}} \ldots x_{n}^{\alpha_{n}}, \partial^{\alpha}=$ $\partial_{1}^{\alpha_{1}} \ldots \partial_{n}^{\alpha_{n}}$ where $\mathbb{N}_{0}$ is the set of non-negative integers and $\partial_{j}=\partial / \partial x_{j}$.

We first introduce the real version of the Fourier hyperfunctions in [KCK].

Definition 2.1. $\quad$ We denote by $\mathcal{F}$ or $\mathcal{F}\left(\mathbb{R}^{n}\right)$ the Sato space of all infinitely differentiable functions $\varphi$ in $\mathbb{R}^{n}$ such that

$$
\|\varphi\|_{h, k}=\sup _{\substack{x \in \mathbb{R}^{n} \\ \alpha \in \mathbb{N}_{0}^{n}}} \frac{\left|\partial^{\alpha} \varphi(x)\right| \exp k|x|}{h^{-|\alpha|} \alpha !}<\infty
$$

for some $h, k>0$.

We say that $\varphi_{j} \rightarrow 0$ as $j \rightarrow \infty$ if $\left\|\varphi_{j}\right\|_{h, k} \rightarrow 0$ as $j \rightarrow \infty$ for some $h, k>0$, and denote by $\mathcal{F}^{\prime}$ the strong dual of $\mathcal{F}$ and call its elements Fourier hyperfunctions.

It is proved in [CCK1] that the inequality (2.1) is equivalent to

$$
\sup _{x \in \mathbb{R}^{n}}|\varphi(x)| \exp k|x|<\infty, \quad \sup _{\xi \in \mathbb{R}^{n}}|\hat{\varphi}(\xi)| \exp h|\xi|<\infty
$$

for some $h, k>0$. 
We now introduce analytic functionals to define hyperfunctions as done by Martineau.

Definition 2.2. $\quad$ Let $K$ be a compact subset of $\mathbb{C}^{n}$. Then we denote by $A^{\prime}(K)$ the space of linear forms $u$ on the space $A$ of entire analytic functions in $\mathbb{C}^{n}$ such that for every neighborhood $\omega$ of $K$ there exists $C_{\omega}>0$ satisfying

$$
|u(\varphi)| \leq C_{\omega} \sup _{\omega}|\varphi|, \quad \varphi \in A .
$$

We call the elements of $A^{\prime}(K)$ analytic functionals carried by $K$.

It follows easily from Pringsheim's theorem the condition (2.3) is equivalent to the condition that for any $h>0$ there exists a positive constant $C$ such that

$$
|u(\varphi)| \leq C \sup _{\substack{x \in K \\ \alpha \in \mathbb{N}_{0}^{n}}} \frac{\left|\partial^{\alpha} \varphi(x)\right|}{h^{-|\alpha|} \alpha !}, \quad \varphi \in A .
$$

We now define the space $\mathcal{B}(\Omega)$ of hyperfunctions following A. Martineau as in $[\mathrm{H}]$.

Definition 2.3. Let $\Omega$ be a bounded open set in $\mathbb{R}^{n}$. Then the space $\mathcal{B}(\Omega)$ of hyperfunctions is defined by $\mathcal{B}(\Omega)=A^{\prime}(\bar{\Omega}) / A^{\prime}(\partial \Omega)$.

By virtue of the localization theorem in $[\mathrm{H}$, Theorem 9.22] we can define hyperfunctions $u \in \mathcal{B}\left(\mathbb{R}^{n}\right)$ as a collection of $u_{j} \in A\left(\bar{\Omega}_{j}\right)$ such that $u_{j}=u_{k}$ in $\Omega_{j} \cap \Omega_{k}, j, k \geq 1$, where $\Omega_{j}, j \geq 1$ is a collection of bounded open sets with $\cup \Omega_{j}=\mathbb{R}^{n}$.

We denote by $E(x, t)$ the $n$-dimensional heat kernel

$$
E(x, t)= \begin{cases}(4 \pi t)^{-n / 2} \exp \left(-|x|^{2} / 4 t\right), & t>0 \\ 0, & t \leq 0\end{cases}
$$

Note that $E(x, t)$ belongs to the space $\mathcal{F}$ for each $t>0$. Thus

$$
U(x, t)=(u * E)(x, t)=u_{y}(E(x-y, t)), \quad x \in \mathbb{R}^{n}, t>0
$$

is a $C^{\infty}$-function in $\mathbb{R}_{+}^{n+1}=\left\{(x, t) \mid x \in \mathbb{R}^{n}, t>0\right\}$ for all $u \in \mathcal{F}^{\prime}$. We call $U(x, t)$ the Gauss transform or defining function of $u$. In Widder[W] it is also called the Poisson transform.

We represent the Fourier hyperfunctions and hyperfunctions as the initial values of solutions of the heat equation. 
Theorem 2.4 [KCK]. Let $u \in \mathcal{F}^{\prime}\left(\mathbb{R}^{n}\right)$. Then the Gauss transform $U(x, t)$ of $u$ is a solution of the heat equation

$$
(\partial / \partial t-\Delta) U(x, t)=0
$$

and satisfies the following growth condition; for every $\varepsilon>0$ there exists a constant $C_{\varepsilon}>0$ such that

$$
|U(x, t)| \leq C_{\varepsilon} \exp [\varepsilon(|x|+1 / t)]
$$

for $t>0, x \in \mathbb{R}^{n}$.

Conversely, let $U(x, t) \in C^{\infty}\left(\mathbb{R}_{0}^{n+1}\right)$ satisfy (2.6) and (2.7). Then there exists a unique $u \in \mathcal{F}^{\prime}\left(\mathbb{R}^{n}\right)$ such that $U(x, t)=u_{y}(E(x-y, t))$.

Theorem 2.5 [M3]. Let $u=\left(u_{j}\right) \in \mathcal{B}\left(\mathbb{R}^{n}\right)$. Then there exists a solution $U(x, t) \in C^{\infty}\left(\mathbb{R}_{+}^{n+1}\right)$ of the heat equation satisfying the following growth conditions; for every compact subset $K \subset \mathbb{R}^{n}$ and for every $\varepsilon>0$ there exists a constant $C_{\varepsilon, K}>0$ such that

$$
|U(x, t)| \leq C_{\varepsilon, K} \exp (\varepsilon / t), \quad t>0, x \in K
$$

and

$$
U(x, t) \rightarrow u \quad \text { as } \quad t \rightarrow 0^{+}
$$

in the sense that $U(x, t)-U_{j}(x, t) \rightarrow 0$ as $t \rightarrow 0^{+}$in $\Omega_{j}, j=1,2, \ldots$, where $U_{j}$ is the Gauss transform of $u_{j}$ defined as in (2.5).

Conversely, let $U(x, t)$ be a solution of the heat equation satisfying the growth condition (2.8). Then there exist a unique $u=\left(u_{j}\right) \in \mathcal{B}\left(\mathbb{R}^{n}\right)$ satisfying (2.9).

Remark 2.6. The Gauss transform $U(x, t)$ of a distribution $u \in \mathcal{D}^{\prime}$ satisfies the growth conditions; for every compact subset $K \subset \mathbb{R}^{n}$ there exist positive constants $C=C(K)$ and $N=N(K)$ such that

$$
|U(x, t)| \leq C t^{-N}, \quad t>0, x \in K
$$

and the Gauss transform $U(x, t)$ of a tempered distribution $u \in \mathcal{S}^{\prime}$ satisfies the growth conditions; there is a positive constants $C, M$ and $N$ such that

$$
|U(x, t)| \leq C(1+|x|)^{M} t^{-N} .
$$

For the other spaces of generalized functions we refer to [CK1, CK2, CL, KCK, M1, M2]. 


\section{$\S 3 . \quad$ Bochner-Schwartz Type Theorem for Hyperfunctions}

Recall that a continuous function $f(x)$ on $\mathbb{R}^{n}$ is positive definite if

$$
\sum_{j, k=1}^{m} f\left(x_{j}-x_{k}\right) \zeta_{j} \overline{\zeta_{k}} \geq 0
$$

for any $x_{1}, x_{2}, \ldots, x_{m} \in \mathbb{R}^{n}$ and any $\zeta_{1}, \zeta_{2}, \ldots, \zeta_{m} \in \mathbb{C}$.

It is well known that the inequality (3.1) is equivalent to

$$
\int \overline{f(x-y)} \varphi(y) \overline{\varphi(x)} d x d y=\left\langle f, \varphi * \varphi^{*}\right\rangle \geq 0
$$

for any $\varphi \in C_{c}^{\infty}$, where $\varphi^{*}(x)=\overline{\varphi(-x)}$.

Thus a distribution (tempered distribution, resp.) $u$ is said to be positive definite if

$$
\left\langle u, \varphi * \varphi^{*}\right\rangle \geq 0
$$

for any test function $\varphi \in C_{c}^{\infty}(\varphi \in \mathcal{S}$, resp).

Generalizing the positive definite (tempered) distributions, conditionally positive definite (tempered) distributions were introduced in [GV] as follows.

Definition 3.1. A (tempered) distribution $u$ is said to be conditionally positive definite of order $s$ if

$$
\left\langle u,(P \varphi) *(P \varphi)^{*}\right\rangle \geq 0
$$

for every $\varphi \in C_{c}^{\infty}(\varphi \in \mathcal{S}$ resp. $)$ and all homogeneous partial differential operators with constant coefficients of order $s$

$$
P=\sum_{|\alpha|=s} a_{\alpha} \partial^{\alpha} .
$$

Positive definite and conditionally positive definite Fourier hyperfunctions can be defined similarly if (3.2) and (3.3) hold true for $\varphi \in \mathcal{F}\left(\mathbb{R}^{n}\right)$. But the definition (3.2) and (3.3) cannot carry over to hyperfunctions which are analytic functionals locally but not globally.

In [CCK2] a positive definite hyperfunction was defined in terms of the corresponding solution of the heat equation and the Bochner-Schwartz theorem for hyperfunction was obtained.

In this section we define a conditionally positive definite hyperfunction and obtain a Bochner-Schwartz type theorem for hyperfunction, which generalizes the Bochner-Schwartz theorem for hyperfunctions in [CCK2]. 
Recall that a hyperfunction $u \in \mathcal{B}\left(\mathbb{R}^{n}\right)$ is said to be positive definite if there exists a Gauss transform $U(x, t)$ of $u$ such that $U(x, t)$ is positive definite for each $t>0$. In accordance with the definition of positive definite hyperfunctions we define a conditionally positive definite hyperfunction.

Definition 3.2. $\quad$ A hyperfunction $u$ is said to be conditionally positive definite if there exists a Gauss transform $U(x, t)$ of $u$ such that $P \bar{P} U(\cdot, t)$ is positive definite for all differential operators $P$ of the form (3.4) and for each $t>0$.

To justify the above definition we state and prove some analogous results in the spaces of distributions, tempered distributions and Fourier hyperfunctions before we state our main result.

Theorem 3.3. The following conditions are equivalent.

(i) $u$ is a conditionally positive definite tempered distribution of order $s$.

(ii) The Gauss transform $U(x, t)$ of $u$ has the property that $P \bar{P} U(\cdot, t)$ is a positive definite function for each $t>0$ and for all differential operators $P$ of the form (3.4).

Proof. (i) $\Rightarrow$ (ii). Let $u$ be a conditionally positive definite tempered distribution of order $s$. Then by definition $P \bar{P} u$ is a positive definite tempered distribution. If we denote by $E_{t}(\cdot)$ the $n$-dimensional heat kernel we have for all $\varphi \in \mathcal{S}$

$$
\begin{aligned}
\left\langle P \bar{P} U(\cdot, t), \varphi * \varphi^{*}\right\rangle & =\left\langle P \bar{P} u * E_{t}, \varphi * \varphi^{*}\right\rangle \\
& =\left\langle P \bar{P} u,\left(\varphi * E_{t / 2}\right) *\left(\varphi * E_{t / 2}\right)^{*}\right\rangle \\
& \geq 0
\end{aligned}
$$

Thus $P \bar{P} U(\cdot, t)$ is a positive definite function for each $t>0$ by the equivalent condition $\left(3.1^{\prime}\right)$.

(ii) $\Rightarrow($ i). If $P \bar{P} U(\cdot, t)$ is a positive definite function we have for all $\varphi \in \mathcal{S}$.

$$
\begin{aligned}
\left\langle P \bar{P} u, \varphi * \varphi^{*}\right\rangle & =\left\langle u,(P \varphi) *(P \varphi)^{*}\right\rangle \\
& =\lim _{t \rightarrow 0^{+}}\left\langle U(\cdot, t),(P \varphi) *(P \varphi)^{*}\right\rangle \\
& =\lim _{t \rightarrow 0^{+}}\left\langle P \bar{P} U(\cdot, t), \varphi * \varphi^{*}\right\rangle \\
& \geq 0 .
\end{aligned}
$$


Thus $P \bar{P} u$ is a positive definite tempered distribution. This completes the proof.

Since every conditionally positive definite distribution of order $s$ is a conditionally positive definite tempered distribution of the same order as stated in Theorem 1.1 we have the following

Theorem 3.4. The following conditions are equivalent.

(i) $u$ is a conditionally positive definite distribution of order $s$.

(ii) There exists a Gauss transform $U(x, t)$ of $u$ such that $U(x, t)$ has the property that $P \bar{P} U(\cdot, t)$ is a positive definite function for each $t>0$ and for all differential operators $P$ of the form (3.4).

By the same method as in Theorem 3.3 we obtain the similar result for Fourier hyperfunctions.

Theorem 3.5. The following conditions are equivalent.

(i) $u$ is a conditionally positive definite Fourier hyperfunction of order s.

(ii) The Gauss transform $U(x, t)$ of $u$ has the property that $P \bar{P} U(\cdot, t)$ is a positive definite function for each $t>0$ and all differential operator $P$ of the form (3.4).

Now we state and prove our main results.

Theorem 3.6. The following conditions are equivalent for $u \in \mathcal{B}\left(\mathbb{R}^{n}\right)$.

(i) $u$ is a conditionally positive definite hyperfunction of order $s$.

(ii) $u$ is a conditionally positive definite Fourier hyperfunction of order s.

Proof. We need to prove only the implication (i) $\Rightarrow($ ii), since (ii) $\Rightarrow(\mathrm{i})$ is trivial. Let $u$ be a positive definite hyperfunction. Then by definition there exists a Gauss transform $U(x, t)$ of $u$ such that $P \bar{P} U(\cdot, t)$ is positive definite for all differential operators $P$ of the form (3.4). Making use of the Taylor theorem we have

$$
U(x, t)=\sum_{|\gamma|<2 s} \frac{\partial^{\gamma} U(0, t)}{\gamma !} x^{\gamma}+\sum_{|\gamma|=2 s} \frac{2 s}{\gamma !} x^{\gamma} \int_{0}^{1}(1-y)^{2 s-1} \partial^{\gamma} U(x y, t) d y
$$


Since the space of hyperfunctions is invariant under differentiation, putting $K=\{0\}$ in (2.8) we have the following estimates for each $|\gamma|<2 s$; for every $\varepsilon>0$, there exists a constant $C_{\varepsilon, \gamma}$ such that

$$
\left|\partial^{\gamma} U(0, t)\right| \leq C_{\varepsilon, \gamma} \exp (\varepsilon / t), \quad t>0 .
$$

To estimate the remainder part of (3.5) we use the fact that every positive definite function attains its maximum at the origin.

For each $|\gamma|=2 s$ we can write $\partial^{\gamma}=\partial^{\alpha} \partial^{\beta},|\alpha|=|\beta|=s$ and

$$
\begin{aligned}
\partial^{\gamma} & =\left(\frac{1}{2} \partial^{\alpha}+\frac{1}{2} \partial^{\beta}\right)^{2}-\left(\frac{1}{2} \partial^{\alpha}-\frac{1}{2} \partial^{\beta}\right)^{2} \\
& =D \bar{D}-D^{*} \bar{D}^{*}
\end{aligned}
$$

where $D=\frac{1}{2} \partial^{\alpha}+\frac{1}{2} \partial^{\beta}, D^{*}=\frac{1}{2} \partial^{\alpha}-\frac{1}{2} \partial^{\beta}$.

Since $D \bar{D} U(\cdot, t)$ and $D^{*} \bar{D}^{*} U(\cdot, t)$ are positive definite functions and attain their maximums at the origin we have

$$
\begin{aligned}
\left|\partial^{\gamma} U(x y, t)\right| & =\left|D \bar{D} U(x y, t)-D^{*} \bar{D}^{*} U(x y, t)\right| \\
& \leq|D \bar{D} U(0, t)|+\left|D^{*} \bar{D}^{*} U(0, t)\right| .
\end{aligned}
$$

Thus by the estimate (2.8) we have the growth condition for each $|\gamma|=2 s$; for every $\varepsilon$ there exists $C_{\varepsilon, \gamma}$ such that

$$
\left|\partial^{\gamma} U(x y, t)\right| \leq C_{\varepsilon, \gamma} \exp (\varepsilon / t) .
$$

It follows that

$$
\begin{aligned}
|U(x, t)| & \leq \sum_{|\gamma|<2 s} \frac{\left|\partial^{\gamma} U(0, t)\right|}{\gamma !}\left|x^{\gamma}\right|+\sum_{|\gamma|=2 s} \frac{2 s}{\gamma !}\left|x^{\gamma}\right| \int_{0}^{1}\left|(1-y)^{2 s-1}\right| \partial^{\gamma} U(x y, t) \mid d y \\
& \leq \exp (\varepsilon / t) \sum_{|\gamma| \leq 2 s} C_{\varepsilon, \gamma}^{\prime}\left|x^{\gamma}\right| \\
& \leq C_{\varepsilon}^{\prime}(1+|x|)^{2 s} \exp (\varepsilon / t) .
\end{aligned}
$$

Thus $U(x, t)$ satisfies the growth condition (2.7) and defines a Fourier hyperfunction $u$.

Remark. The proof of this main theorem is much subtler than the proof for the Bochner-Schwartz theorem for positive definite hyperfunctions in [CCK2].

Combining Theorem 3.3 and Theorem 3.4 we have the following result. 
Corollary 3.7. The following conditions are equivalent.

(i) $u$ is a conditionally positive definite distribution of order $s$.

(ii) $u$ is a conditionally positive definite tempered distribution of order $s$.

(iii) The Gauss transform $U(x, t)$ of u satisfies the growth condition; there exist positive constants $C$ and $N$ such that

$$
|U(x, t)| \leq C(1+|x|)^{2 s} t^{-N}
$$

and $P \bar{P} U(\cdot, t)$ is positive definite function for each $t>0$ and all differential operators $P$ of the form (3.4).

(iv) $u$ can be expressed as in (1.2) of Theorem 1.1.

Proof. The equivalence of the statements (i) and (iv) is just Theorem 1.1 and the implication (iii) $\Rightarrow$ (ii) follows from the Remark 2.6 and Theorem 3.3. Thus it suffices to prove the implication (i) $\Rightarrow$ (iii). Let $u$ be a conditionally positive definite distribution of order $s$. Then by Theorem 3.4 there exists a Gauss transform $U(x, t)$ of $u$ such that $P \bar{P} U(\cdot, t)$ is positive definite for each $t>0$ and for all differential operator $D$ of the form (3.4). Making use of the estimate (2.10) we have for each $\gamma \in \mathbb{N}_{0}^{n}$

$$
\left|\partial^{\gamma} U(0, t)\right| \leq C_{\gamma} t^{-N_{\gamma}}, \quad t>0
$$

Applying the same method as in the proof of Theorem 3.6 with the estimate (3.8) instead of (3.6) we obtain the estimate (3.7).

Combining Theorem 3.5, Theorem 3.6 we have the following.

Corollary 3.8. The following conditions are equivalent

(i) $u$ is a conditionally positive definite hyperfunction of order $s$.

(ii) $u$ is a conditionally positive definite Fourier hyperfunction of order s.

(iii) The Gauss transform $U(x, t)$ of u satisfies the following growth condition; for every $\varepsilon>0$, there exists a constant $C_{\varepsilon}$ such that

$$
|U(x, t)| \leq C_{\varepsilon}(1+|x|)^{2 s} \exp (\varepsilon / t)
$$

and $P \bar{P} U(\cdot, t)$ is positive definite function for each $t>0$ and all differential operators $P$ of the form (3.4). 
(iv) $u$ can be expressed as in (1.2) where the measure $\mu$ in (1.2) satisfies the growth conditions (1.5) and (1.6) and $\alpha(x)$ in (1.2) is given by (1.7).

Putting $s=0$ in the Corollary 3.7 and Corollary 3.8 we have the following Bochner Schwartz theorem for (tempered) distributions and (Fourier) hyperfunctions, respectively.

Corollary 3.9. The following conditions are equivalent.

(i) $u$ is a positive definite (tempered) distribution.

(ii) The Gauss transform $U(x, t)$ of $u$ is a positive definite function and satisfies the growth condition; there exist positive constants $C$ and $N$ such that

$$
|U(x, t)| \leq C t^{-N}
$$

(iii) $u$ is the Fourier transform of a positive tempered measure.

Corollary 3.10. The following conditions are equivalent for $u \in \mathcal{B}\left(\mathbb{R}^{n}\right)$

(i) $u$ is a positive definite (Fourier) hyperfunction

(ii) The Gauss transform $U(x, t)$ of $u$ is a positive definite function satisfying the growth condition; for every $\varepsilon>0$, there exists $C_{\varepsilon}$ such that

$$
|U(x, t)| \leq C_{\varepsilon} \exp (\varepsilon / t)
$$

(iii) $u$ is the Fourier transform of a positive measure of infra-exponential type.

\section{Acknowledgement}

We would like to express our sincere gratitude to the referee for helpful comments. This work was supported by Korea Research Foundation Grant (KRF-99-015-DP0017).

\section{References}

[CCK1] Chung, J., Chung, S.-Y. and Kim, D., A characterization for Fourier hyperfunctions, Publ. RIMS, Kyoto Univ., 30 (1994), 203-208.

[CCK2] _ Positive definite hyperfunctions, Nagoya Math. J., 140 (1995), 139-149.

[CCK3] , Bochner-Schwartz type theorem for conditionally positive definite Fourier hyperfunctions, Positivity, 7 (2003), 323-334.

[CK1] Chung, S.-Y. and Kim, D., Distributions with exponential growth and BochnerSchwartz theorem for Fourier hyperfunctions, Publ. RIMS, Kyoto Univ., 31 (1995), 829-845. 
[CK2] Chung, S.-Y. and Kim, D., Representation of quasianalytic ultradistributions, Ark. Mat., 31 (1993), 51-60.

[CL] Chung, J. and Lee, S. J., Representation of the generalized functions of Gelfand and Shilov, Commun. Korean Math. Soc., 9 (1994), 607-616.

[GHS] Guo, K., Hu, S. and Sun, X., Conditionally positive definite functions and LaplaceStieltjes integrals, J. Approx. Theory, 74 (1993), 249-265.

[GV] Gelfand, I. M. and Vilenkin, N. Y., Generalized Functions IV, Academic Press, New York, 1964.

[H] Hörmander, L., The analysis of linear partial differential operators I, SpringerVerlag, Berlin-New York, 1983.

[KCK] Kim, K. H., Chung, S.-Y. and Kim, D., Fourier hyperfunctions as the boundary values of smooth solutions of heat equations, Publ. RIMS, Kyoto Univ., 29 (1993), 289-300.

[M1] Matsuzawa, T., A calculus approach to hyperfunctions II, Trans. Amer. Math. Soc., 313 (1990), 619-654.

[M2] , A calculus approach to hyperfunctions III, Nagoya Math. J., 118 (1990), 133-153.

[MN] Madych, W. R. and Nelson, S. A., Multivariate interpolation and conditionally positive definite functions II, Math. Comput., 54 (1990), 211-230.

[S] Schwartz, L., Théorie des distributions, Hermann, Paris, 1966.

[W] Widder, D. V., Heat equation, Academic Press, New York, 1975.

[Z] Zoltán Sasvári, Positive definite and definitizable functions, Akademie Verlag, Germany, 1994. 